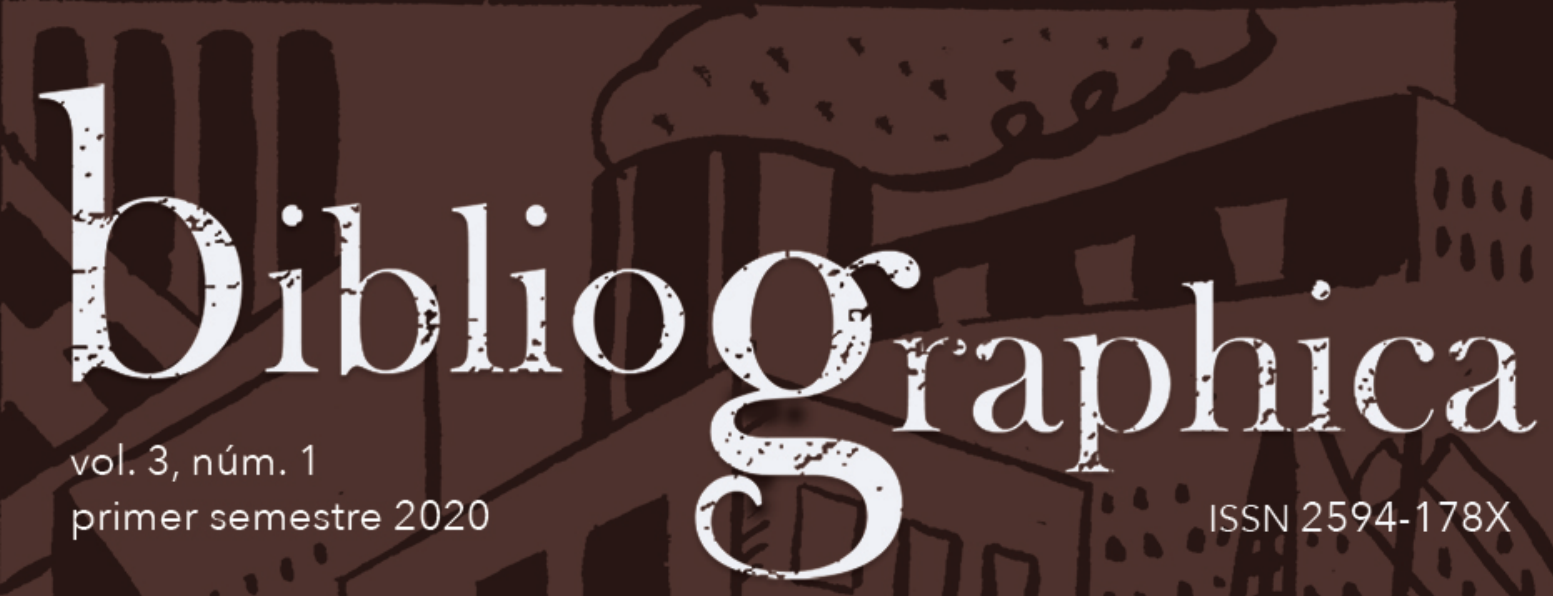




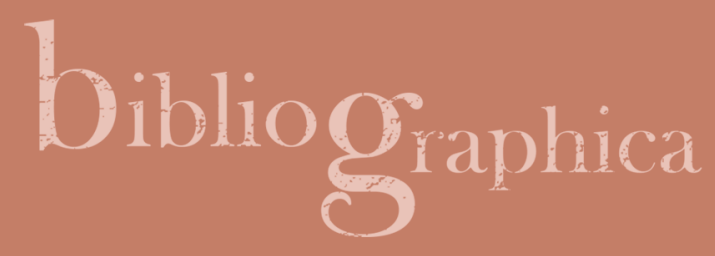

\section{Organización del archivo Grupo de Información en Reproducción Elegida (GIRE)}

Organizing the Information Group

on Elected Reproduction Archive

\section{Ángel Ángeles Fernández}

angelfer2006@hotmail.com

Universidad Nacional Autónoma de México

Archivo Histórico

Instituto de Investigaciones sobre la Universidad y la Educación

Recepción: 22.04.2019 / Aceptación: 10.06.2019

DOI: https://doi.org/10.22201/iib.2594178xe.2020.1.46 
Resumen Este artículo aborda la manera en que se ha trabajado la organización archivística del fondo documental del Grupo de Información en Reproducción Elegida (GIRE), resguardado en el Archivo Histórico de la Universidad Nacional Autónoma de México (AHUNAM). El método para dicha organización consta de seis etapas: identificación, clasificación, ordenación, guarda, foliación y descripción, con el propósito de que pueda ser consultado por los usuarios. Además, da cuenta del tipo de material y contenido temático que integra este fondo documental. En la actualidad ya está ordenado y las seis etapas mencionadas son analizadas en este trabajo; por otra parte, el fondo documental cuenta con un catálogo por expediente.

Palabras clave

Abstract

Keywords
Archivística; archivos; fondo documental; organización; GIRE.

This article describes the working approach and organization system used for the Information Group on Elected Reproduction archive preserved at the Historical Archive of the National Autonomous University of Mexico (AHUNAM). The method for that organization consists of six stages: identifying, classifying, ordering, filing, numbering and describing, so the users can access information provided by the fund. Moreover, the article accounts for the type of material and the thematic content integrating this documentary fund. The Information Group on Elected Reproduction archive is currently systematically arranged, which entails the coverage of the six steps mentioned that will be analyzed in this article. Each of the files integrating the fund has already been catalogued.

Archival science; archives; documentary fund; organization; GIRE. 


\section{Introducción}

Este trabajo aborda el método con el cual se ha trabajado el material que conforma el fondo denominado Grupo de Información en Reproducción Elegida (GIRE), resguardado por el Archivo Histórico de la Universidad Nacional Autónoma de México (AHUNAM). La finalidad es rescatar el conocimiento adquirido, la experiencia profesional al organizar un archivo y compartir los procedimientos de organización y descripción de este fondo documental, al igual que explicar cómo se elaboraron sus instrumentos de consulta: guías, cuadro de clasificación, inventario, catálogo por expediente y catálogo por documento.

Se ha tomado en consideración lo que menciona la Ley General de Archivos sobre la Organización, que es el conjunto de operaciones intelectuales y mecánicas destinadas a la clasificación, ordenación y descripción de los diferentes grupos documentales, con el propósito de consultar y recuperar la información. ${ }^{1}$ Lo anterior permitirá al investigador, estudiante y público en general consultar adecuadamente el material documental del GIRE.

El trabajo archivístico tiene como cometido que un fondo o colección esté completamente identificado, clasificado, ordenado, resguardado, foliado y descrito; y que estas seis etapas queden reflejadas en un instrumento descriptivo, el cual permita la pronta localización del documento de interés para el usuario. Para lograr que el trabajo sea óptimo debe pasar por varios procesos, como lo menciona el "Manual de procedimientos técnicos del Archivo Histórico de la UNAM": ${ }^{2}$ identificación, organización, descripción, restauración, reprografía y difusión. En este artículo nos centraremos en los tres primeros procedimientos, los cuales se llevaron a cabo durante tres años de trabajo.

Se mostrará qué es y cómo se conformó el fondo GIRE, cuándo ingresó al AHUNAM, cómo se llevaron a cabo las seis etapas mencionadas para organizar, cuidar, preservar y describir el material que reunió un grupo de feministas.

\footnotetext{
1 Instituto Nacional de Transparencia, Acceso a la Información y Protección de Datos Personales, Ley General de Archivos (México: INAI, 2019), 21.

2 El "Manual de procedimientos técnicos del Archivo Histórico de la UNAM" es un material inédito, coordinado por Georgina Flores Padilla, se trata de un documento que no ha sido aprobado por el Consejo Interno del Instituto de Investigaciones sobre la Universidad y la Educación (IISUE), pero resulta una referencia fundamental para el trabajo archivístico realizado en el AHUNAM.
} 


\section{Grupo de Información en Reproducción Elegida (GIRE)}

El GIRE es una asociación civil sin fines de lucro fundada en abril de 1992 por Marta Lamas, Patricia Mercado, María Consuelo Mejía, Sara Sefchovich y Lucero González. Su principal interés es informar acerca de la salud reproductiva y defender los derechos reproductivos de las mujeres en el marco de los derechos humanos. ${ }^{3}$

Se trata de una organización interesada en el tema de los derechos reproductivos, para quienes toman decisiones como líderes de opinión y profesionales de derecho y salud. Desde sus orígenes el GIRE recopila, sistematiza y difunde información sobre derechos reproductivos, y trabaja para que todas las personas en México puedan ejercer su derecho a decidir acerca de su propia reproducción. Su labor se centra en promover reformas legales y políticas públicas que amplíen y garanticen el acceso a los servicios de salud reproductiva y al ejercicio de los derechos correspondientes.

Su trabajo está dirigido a quienes toman decisiones, por ejemplo funcionarios de los poderes Ejecutivo, Legislativo y Judicial; profesionales del derecho, personal relacionado con la procuración de justicia, prestadores de servicios de salud, medios de comunicación, líderes de opinión, personal académico, organizaciones de la sociedad civil y público en general. En la actualidad se enfoca en el tema del aborto y de los derechos reproductivos, a partir de la perspectiva de los derechos humanos y laicidad del Estado. Desde su fundación el GIRE también trabaja para hacer avanzar las leyes y políticas públicas sobre el aborto en México, así como el acceso a servicios de salud de calidad. ${ }^{4}$

\section{Ingreso del fondo GIRE al Archivo Histórico de la UNAM}

El fondo documental GIRE fue donado y se propuso conservarlo en una sola unidad, en un lugar adecuado, que fuera organizado y descrito apropiadamente, además de tomar las medidas adecuadas para evitar su deterioro. Firmó el acuerdo el 29 de abril del 2016 la maestra Regina Tamés Noriega, directora del GIRE, en un oficio dirigido al doctor Mario Rueda Beltrán, director del Instituto

\footnotetext{
${ }^{3}$ GIRE, Boletín Trimestral sobre Reproducción Elegida, núm. 1 (abril de 1994): 1.

${ }^{4}$ GIRE, "¿Quiénes somos?", acceso el 2 de diciembre de 2019, https://www.gire.org.mx/ quienes-somos.
} 
de Investigaciones sobre la Universidad y la Educación (IISUE), con copia para la coordinadora general del AHUNAM, doctora Clara Inés Ramírez. ${ }^{5}$

Ingresó el fondo GIRE al AHUNAM el 2 de mayo de 2016 y fue recibido por la doctora Ramírez. ${ }^{6}$ Estaba resguardado en 53 cajas AM-70 y 2 cajas LF-50 de polipropileno.7 GIRE es una asociación joven, por tanto sólo fue entregada la documentación correspondiente al área de Biblioteca, pero no donaron videos, libros ni bases de datos que pertenecen a la misma área.

El fondo contiene artículos mecanuscritos, manuscritos, recortes y fotocopias hemerográficas, colección de folletos y de objetos, material didáctico, una colección de 482 carteles, 15 fotografías y 558 títulos de revistas sobre feminismo y estudios de género. Los años extremos que abarca van de 1934 a 2015, predomina la documentación de 1980 a 2015. En la actualidad son 212 cajas de polipropileno, ${ }^{8} 23.4$ metros lineales que resguardan 1003 expedientes en soportes impresos, CD, USB, objetos y material didáctico.

Al ingresar al AHUNAM, el fondo no presentaba ningún trabajo archivístico previo. Primero, el Departamento de Restauración le hizo un diagnóstico y se elaboró su expediente técnico. El 14 de noviembre de 2016 me asignaron el fondo GIRE para su organización, y llevé a cabo el método mencionado de identificación, clasificación, ordenación, guarda, foliación y descripción. ${ }^{9}$ Considerando la Ley General de Archivos, estamos obligados a adoptar las medidas y procedimientos que garanticen la conservación de la información, independientemente de su soporte documental. ${ }^{10}$

El fondo GIRE cuenta actualmente con un catálogo por expediente, un catálogo por documento de las cajas 202 a 212 y una relación de todo el fondo documental. A continuación explico cada una de las etapas, así como los productos obtenidos.

\footnotetext{
${ }^{5}$ Oficio dirigido al director del IISUE, dr. Mario Rueda Beltrán, firmado por la mtra. Regina Tamés Noriega, directora del GIRE, con fecha del 29 de abril de 2016.

${ }^{6}$ Oficio de aceptación del fondo documental GIRE, firmado por la dra. Clara Ramírez, coordinadora AHUNAM-IISUE, con fecha del 2 de mayo de 2016.

${ }^{7}$ Cajas de archivo muerto de $70 \times 40 \mathrm{~cm}$ y cajas de $50 \times 40 \mathrm{~cm}$., respectivamente, donde se resguardan los carteles.

${ }^{8} 208$ cajas AG-12 de $12 \times 40 \mathrm{~cm}$ y 4 cajas LF- 50 de $50 \times 40 \mathrm{~cm}$, donde se resguardan los materiales.

9 Mariano Mercado Estrada, "Guía del fondo Heberto Castillo Martínez", en Teoría y práctica archivística III, coord. de Mariano Mercado Estrada (México: UNAM, CESU, 2003), 56.

${ }^{10}$ INAI, Ley General de Archivos, 61.
} 


\section{La identificación}

"Guía del orden en el cual ingresó el fondo del GIRE al AHUNAM"

La primera etapa fue la identificación de los materiales que conforman el fondo GIRE. Inicié con el cambio de las cajas AM-70 a cajas AG-12 de cartón, respetando el orden de origen de los documentos. ${ }^{11}$ Es importante mencionar que no se trató de ordenar los materiales, sólo se cambiaron guardas para que no se dañaran y pudieran manipularse en las etapas de ordenación y resguardo.

Preparé las cajas de cartón AG-12, cada una con una etiqueta colocada en un extremo, que indica el nombre del fondo, "Grupo de Información en Reproducción Elegida GIRE", y un número consecutivo (hasta el 229 en cajas). Luego enumeré las carpetas ${ }^{12}$ donde temporalmente quedaron resguardados los documentos.

Consulté el "Manual de procedimientos técnicos del Archivo Histórico de la UNAM", el cual considera que la identificación en los archivos de este tipo reside en analizar las características formales e informativas de los documentos de un fondo o colección, y el contexto histórico administrativo en que fueron producidos, con el fin de establecer las series documentales. ${ }^{13}$

En la etapa de identificación, el fondo documental GIRE fue depositado temporalmente en guardas de primer nivel ( 2180 carpetas) y en guardas de segundo nivel, en 225 cajas AG-12 de cartón y 4 LF-50, que sumaron en total 229 cajas, para su identificación y clasificación. Al efectuar este cambio de cajas, se realizó una revisión exhaustiva del material, en cuanto a los soportes y las temáticas, para conformar las Secciones y las Series. Cabe reiterar que fue respetado el orden original de los documentos, tal y como llegaron al AHUNAM.

Es importante mencionar que la diferencia de este método de organización radica en la elaboración de la "Guía del orden en el cual ingresó el fondo GIRE al AHUNAM", ${ }^{14}$ porque esta guía no la hacen en ningún otro método y es fundamental para dejar constancia de cómo se encontraba el material a su ingreso

\footnotetext{
${ }^{11}$ Uno de los principios fundamentales en la archivística: el Respeto al Orden Original. Los materiales no se mezclan.

${ }^{12}$ Carpetas es un término utilizado en archivística, son fólderes tamaño oficio, marcados con un número consecutivo y otros datos importantes de los documentos: año, número de documentos y de fojas.

${ }^{13}$ Flores Padilla, "Manual de procedimientos...", 48.

${ }^{14}$ Éste es el primer instrumento de consulta, el producto que resulta de la identificación.
} 
al archivo. Es más completa que un inventario y es el primer instrumento de consulta: al elaborarla ya se puede dar servicio a los usuarios.

Para elaborarla fue necesario hacer también fichas catalográficas. El modelo de la ficha fue tomado de Teoría y práctica archivística $V I{ }^{15}$ que tiene relación con la ficha mencionada en el Manual de procedimientos técnicos para archivos históricos de universidades e instituciones de educación superior. ${ }^{16}$

También se tomaron en consideración las reglas internacionales, como la Norma Internacional General de Descripción Archivística ISAD(G), adaptada por el Comité de Normas de Descripción en Estocolmo, Suecia, en septiembre de 1999. Es una guía general para elaborar descripciones archivísticas, identificar y explicar el contexto y contenido de los documentos en los archivos, con el propósito de hacerlos accesibles: "Las reglas identifican y definen 26 elementos que pueden combinarse entre sí para constituir la descripción de una entidad archivística. La estructura y el contenido de la información de cada uno de estos elementos deberán formularse de acuerdo con las normas nacionales aplicables en cada país". 17

Como mencioné, para elaborar la "Guía del orden...", en donde se registra el número de documentos, de fojas, los años extremos y el contenido temático, fue necesario preparar fichas catalográficas, cuyos elementos presento enseguida.

${ }^{15}$ María Sandra Mondragón Aguilar, "Descripción de la Correspondencia de las Obras Literarias del Fondo Francisco L. Urquizo". En Teoría y práctica archivística VI, coord. de Gustavo Villanueva, Cuadernos del Archivo Histórico de la UNAM 22 (México: UNAM, IISUE, 2012), 102.

${ }^{16}$ Los datos básicos de las fichas catalográficas son: número progresivo de ficha, fecha completa (año, mes, día), lugar(es) de emisión del documento, entrada descriptiva, observaciones, ubicación archivística del documento: siglas del fondo, sección, subsección o serie; ubicación topográfica: número de caja, número de expediente, número de documentos y fojas. Gustavo Villanueva Bazán, coord., Manual de procedimientos técnicos para archivos históricos de universidades e instituciones de educación superior (México: UNAM, CESU / AHBUAP, 2002), 93.

${ }_{17}$ ISAD(G): Los elementos de la descripción se dividen en las siguientes áreas: de identificación, de contexto, de contenido y estructura, de condiciones de acceso y uso, de documentación asociada, de notas y de control de la descripción. Para consultar cada uno de los 26 elementos más detenidamente, véase Norma Internacional General de Descripción Archivística ISAD(G) (Madrid: Consejo Internacional de Archivos, 2000), acceso el 2 de diciembre de 2019, https://www.ica.org/sites/default/files/isad\%20g\%20SP.pdf. 


\section{Artículos, folletos y hemerografía:}

1. Carpeta

2. Años

3. Documentos

4. Fojas

5. Tipo de material

6. Contenido

7. Observaciones

8. Caja

Revistas:

1. Carpeta

2. Años

3. Núm. de fascículos

4. Fojas

5. Título

6. ISSN

7. País

8. Observaciones

9. Caja

\section{Objetos:}
1. Carpeta
2. Título
3. Imagen
4. Año
5. Descripción
6. Observaciones
7. Fondo
8. Núm. de caja
9. Núm. de piezas 
Por otra parte, se digitalizó el material con el propósito de ilustrar la ficha de los objetos y carteles.

\section{Carteles:}

1. Carpeta

2. Título

3. Imagen

4. Año

5. Descripción

6. Observaciones

7. Medidas

8. Fondo

9. Núm. de caja

10. Núm. de ejemplares

Las fichas catalográficas fueron de utilidad para elaborar el primer instrumento de consulta y producto de la identificación, la "Guía del orden....,"18 que sirvió para trazar el plan de la clasificación.

En el "Manual de procedimientos técnicos del Archivo Histórico de la UNAM"no se menciona un instrumento así, pero resulta fundamental para ejecutar una revisión y registro de los materiales con el propósito de tener una visión integral del estado del grupo documental a su llegada; asimismo, permite realizar cualquier otro instrumento de consulta y concluir satisfactoriamente la etapa de la identificación.

Con este instrumento del fondo documental GIRE la etapa de identificación concluyó en ocho meses, y el fondo ya podía consultarse. Al año de iniciar este procedimiento se realizó su primera consulta: una usuaria que cursaba su doctorado en derecho por la Universidad de Harvard en Cambridge, Massachusetts, Estados Unidos.

\footnotetext{
${ }_{18}$ Ángel Ángeles Fernández, "Guía del orden en el cual ingresó el fondo Grupo de Información en Reproducción Elegida al AHUNAM", 4 vols. (México: UNAM, IISUE, AHUNAM, 2017), 1493. (Inédito).
} 


\section{La clasificación}

José Ramón Cruz Mundet reflexiona acerca de que la clasificación consiste en agrupar jerárquicamente los documentos de un fondo mediante clases, de lo general a lo particular, según los principios de procedencia y el orden original. ${ }^{19}$ Describe en su Manual de archivística los tres elementos de clasificación, que son:

1. La clasificación funcional, realizada a partir de las funciones de la entidad que generó el fondo documental, las cuales serán tomadas en consideración para clasificar, es decir que se agrupará de acuerdo con las actividades que lleva a cabo la entidad.

2. La clasificación orgánica, sistema donde las series se agrupan de acuerdo con las diferentes divisiones administrativas o estructura orgánica de la entidad, reproduciendo sus servicios, secciones o unidades, o sea, según la actividad que realizan en la entidad.

3. La clasificación por material es resultado del análisis del contenido de los documentos, de los asuntos o materias sobre los que tratan, que podrían ser clasificados por títulos. ${ }^{20}$

Considerando lo anterior, la mejor manera de ordenar el fondo documental GIRE fue la clasificación por material, un mecanismo de gran ayuda para organizar el fondo de manera correcta y funcional. Como resultado de esta etapa se elaboró un cuadro de clasificación, como lo menciona Mariano Mercado, un mapa conceptual que permite ordenar el fondo en su totalidad. ${ }^{21}$ En otras palabras, se identificaron las secciones y las series. En el Instructivo para elaborar el Cuadro general de clasificación archivística, el Archivo General de la Nación menciona que:

La estructura del Cuadro general de clasificación archivística será jerárquica y atenderá los conceptos de fondo, sección y serie que establecen un principio de diferenciación y estratificación de las diversas agrupaciones documentales

\footnotetext{
19 José Ramón Cruz Mundet, Manual de archivística (España: Fundación Germán Sánchez Ruipérez, 2003), 240.

20 lbid., 240-242.

${ }^{21}$ Mercado, "Guía del fondo Heberto Castillo...", 55.
} 
que conforman el acervo de una dependencia o entidad. De esta manera, los documentos se reúnen en agrupaciones naturales, a saber: fondo, sección, serie (subserie), expediente, unidad documental. ${ }^{22}$

El cuadro de clasificación del fondo GIRE es el siguiente:

1. Área de Comunicación

1.1 Artículos acerca del aborto

1.2 Artículos acerca de maternidad y paternidad

1.3 Artículos acerca de reproducción humana

1.4 Artículos acerca de derechos humanos

1.5 Artículos acerca de género

1.6 Artículos acerca de métodos anticonceptivos

1.7 Artículos acerca de conferencias, declaraciones internacionales y encuentros

1.8 Artículos acerca de organizaciones no gubernamentales (ONG's), entrevistas, conferencias e invitaciones

1.9 Artículos acerca de violencia en contra de la mujer

1.10 Artículos acerca de población y desarrollo

1.11 Artículos acerca del síndrome de inmunodeficiencia adquirida (VIH-SIDA)

1.12 Artículos acerca de salud reproductiva

1.13 Artículos acerca de discriminación de la mujer y feminismo

1.14 Artículos acerca de religión

1.15 Artículos acerca de infancia y adolescencia

1.16 Artículos acerca de derechos sexuales y reproductivos

1.17 Artículos acerca de muerte materna

1.18 Artículos acerca de católicas por el derecho a decidir

1.19 Artículos acerca de reproducción asistida

1.20 Artículos de Marta Lamas

1.21 Artículos de Edgar González Ruiz

1.22 Artículos de María Consuelo Mejía

1.23 Ponencias

1.24 Encuestas y estadísticas

${ }^{22}$ AGN, Instructivo para elaborar el Cuadro general de clasificación archivística (México: AGN, 2012), 10. 
1.25 Artículos acerca de anticonceptivos de emergencia

1.26 Informes

1.27 Artículos acerca de la salud de la mujer

1.28 Artículos acerca de la interrupción voluntaria y legal del embarazo

1.29 Artículos acerca de la violencia intrafamiliar y feminicidios

1.30 Artículos acerca de planificación familiar

1.31 Carpetas y hojas informativas

1.32 Notas hemerográficas, agencias, radio y televisión

2. Desarrollo Institucional

2.1 Documentos acerca del Grupo de Información en Reproducción Elegida (GIRE)

2.2 Correspondencia

3. Litigio e Incidencia Política

3.1 Caso de Paulina Ramírez Jacinto

3.2 Iniciativas de reformas sobre el aborto y despenalización

3.3 Recomendaciones, pronunciamientos, reformas de ley y declaraciones

3.4 Prestación ineficiente en agencias especializadas, decretos e iniciativas sobre maternidad voluntaria

3.5 Diario oficial y gaceta oficial

4. Colección de Folletos

4.1 Países Alemania, Argentina, Australia, Austria

4.2 Países Bangladesh, Bélgica, Belice, Bolivia, Bruselas, Bulgaria

4.3 Países Canadá, Chile, China, Colombia, Costa Rica, Croacia, Cuba

4.4 Países Dinamarca, Ecuador, Egipto, El Salvador, España, Estados Unidos, Europa-Asia (se respetó la carpeta de origen)

4.5 Países Finlandia, Francia, Guatemala, Honduras

4.6 Países India, Inglaterra, Italia

4.7 Países Kenia, Lituania, Malasia, Marruecos, Nicaragua, Nigeria

4.8 Países Paraguay, Países Bajos, Panamá, Philippines, Polonia, Puerto Rico, República Dominicana, Perú 
4.9 Países Scotland, Senegal, Sudáfrica, Suiza

4.10 Países Tailandia, Trinidad y Tobago, Turquía, Ucrania, Uganda, Uruguay, Venezuela

4.11 Estados de Aguascalientes, Baja California Norte, Baja California Sur, Chiapas, Chihuahua, Colima

4.12 Estados de Guanajuato, Estado de México, Hidalgo, Jalisco

4.13 Estado de Morelos, México D. F., Nuevo León, Oaxaca

4.14 Estado de Puebla, Querétaro, San Luis Potosí, Sonora, Tlaxcala, Veracruz, Yucatán, Zacatecas

4.15 Adolescencia e infancia

4.16 Derechos humanos

4.17 Salud sexual

4.18 Infecciones de transmisión sexual

4.19 Métodos anticonceptivos

4.20 Salud de las mujeres

4.21 Derechos sexuales y reproductivos

4.22 Aborto

4.23 Píldora de emergencia

4.24 Maternidad

$4.25 \mathrm{VIH}-\mathrm{SIDA}$

4.26 Población y desarrollo

4.27 Violencia contra la mujer

4.28 Género y equidad

4.29 Diversidad sexual

4.30 Masculinidad

4.31 Gestogramas

4.32 Calcomanías

5. Hemerografía

5.1 Artículos acerca del aborto

5.2 Artículos acerca de coyuntura Chiapas, ley que despenaliza el aborto

5.3 Desplegados y comunicados

5.4 Copias de revistas y libros

5.5 Historietas

5.6 Revistas 
6. Colección de Objetos
6.1 Prendas
6.2 Botones, pluma y pulsera
6.3 Material didáctico, CD y USB

7. Colección de Carteles

\subsection{Carteles acerca del aborto}

7.2 Carteles acerca de mujeres

7.3 Carteles acerca de maternidad

7.4 Carteles acerca de la familia y violencia intrafamiliar

7.5 Carteles acerca de violencia en contra de la mujer

7.6 Carteles acerca de salud de las mujeres, salud reproductiva

7.7 Carteles acerca de planificación familiar

7.8 Carteles acerca de convocatorias, congresos, seminarios, conferencias, diplomados

7.9 Carteles acerca de métodos anticonceptivos

7.10 Carteles acerca de derechos reproductivos y sexuales

7.11 Carteles acerca de población y desarrollo

7.12 Carteles acerca de infancia y jóvenes

7.13 Carteles acerca del derecho a decidir

7.14 Carteles acerca de diversidad sexual

7.15 Carteles acerca de derechos humanos

7.16 Carteles acerca del feminismo

7.17 Carteles acerca del Grupo de Información en Reproducción Elegida (GIRE)

7.18 Carteles acerca de equidad

7.19 Carteles acerca de la píldora de emergencia

7.20 Carteles acerca del VIH/SIDA

7.21 Carteles acerca de mortalidad materna

Con el cuadro de clasificación obtenemos una visión general del fondo documental, ya que presenta las temáticas, las cuales son las líneas de investigación que puede abordar el investigador, estudiante o persona interesada en el tema. La clasificación es de gran ayuda para llevar a cabo las siguientes 
etapas; si ya se identificó y clasificó la documentación, puede continuarse con la ordenación y guarda.

Otra característica sobresaliente del método es que no se separan los materiales en la etapa de clasificación. Este cuadro -que permite mover los documentos a partir de las secciones y series, indicadas en él mismo- resulta fundamental para las dos etapas siguientes.

\section{Ordenación y guarda}

Para la aplicación del cuadro de clasificación a la "Guía del orden..." y la "Guía específica..." retomamos el Manual de archivística de Cruz Mundet, que menciona lo siguiente sobre la organización y descripción de los fondos documentales y colecciones que conforman un archivo:

Organizar el fondo de un archivo consiste en dotarlo de una estructura que reproduzca el proceso mediante el cual los documentos han sido creados. Pero además, atendiendo a la función primordial, nos referimos a la informativa, debe facilitar la localización de los documentos, proporcionando con su estructura organizativa la información suficiente para orientar las búsquedas con acierto, con exclusividad, sin ambigüedades de ningún género; es decir, todas y cada una de las unidades archivísticas, todas y cada una de las series que lo integran, tienen asignada una ubicación conceptual posible y no otra, de manera que la interrogación al esquema organizativo de un fondo documental siempre encontrará respuesta cabal, siguiendo un camino lógico dentro del mismo y sólo uno, sin dar lugar a la ambigüedad ni a la disyuntiva. ${ }^{23}$

De acuerdo con la teoría de Cruz Mundet sobre la organización de un archivo, continuamos con las etapas de la ordenación y guarda. Se aplicó el cuadro de clasificación a la "Guía del orden..." al marcar con lápiz el número de sección y serie registrado en el cuadro de clasificación: 1.1 Artículos acerca del aborto, marcando todo el material relacionado con ese tema; consecutivamente hice lo mismo con las demás series. Recordemos que la "Guía del orden..." está conformada por fichas catalográficas; el marcaje fue aplicado en todo el instrumento de consulta.

\footnotetext{
${ }^{23}$ Cruz Mundet, Manual de archivística, 229.
} 
Con este procedimiento pueden moverse los documentos de manera sistemática, lo cual agiliza la etapa de ordenación. Al terminar el marcaje de toda la "Guía del orden..." los materiales pueden ser movidos, pero antes es necesario hacer las guardas de primer nivel, preparar los expedientes en las carpetas de tres solapas ${ }^{24}$ con numeración consecutiva y las guardas de segundo nivel, es decir las cajas de polipropileno AG-12, que también fueron numeradas consecutivamente. Al conformar los expedientes por sección y serie, los documentos se ordenaron cronológicamente del año más antiguo al más reciente.

La ventaja que tiene este sistema de organización de un fondo documental es que se va moviendo la documentación poco a poco; al mover cada temática-que finalmente representa una serie-, por ejemplo: 1.1 Artículos acerca del aborto, se reunieron los artículos relacionados con ese tema y fueron puestos en las carpetas de tres solapas numeradas consecutivamente, hasta resguardar todos los documentos de esa serie.

A continuación, para proteger los expedientes se colocan en las guardas de segundo nivel o cajas de polipropileno AG-12, también con numeración consecutiva. Al término, se procede con la siguiente serie, en este ejemplo: 1.2 Artículos acerca de maternidad y paternidad. Se repite el mismo procedimiento hasta terminar con todo el material del fondo GIRE.

Al concluir las etapas de ordenación y guarda, los resultados son los siguientes: los años extremos del fondo documental son 1934 y 2015, y predomina la documentación de 1980 a 2015. Actualmente son 212 cajas de polipropileno, 23.4 metros lineales que resguardan 1003 expedientes.

\section{Guía específica}

Cruz Mundet menciona que las guías "proporcionan información sobre todos o parte de los fondos de uno o más archivos, describen globalmente las grandes agrupaciones documentales, esbozan la historia de los organismos productores y facilitan información auxiliar acerca del archivo o archivos y los servicios disponibles". ${ }^{25}$ Estos instrumentos de consulta pueden ser generales o específicos: "Las guías generales [...] dan información global de los fondos y co-

\footnotetext{
${ }^{24}$ Se trata de carpetas tamaño oficio con tres pestañas que permiten "abrazar" los documentos; tienen una pestaña a la derecha e izquierda, así como una inferior, para proteger perfectamente al cerrar cada una de ellas. Este procedimiento sirve para conformar los expedientes por serie.

${ }^{25}$ Cruz Mundet, Manual de archivística, 273.
} 
lecciones que guarda el archivo", mientras que las específicas proporcionan "información de manera exclusiva sobre el contenido de un fondo o colección". ${ }^{26}$

La "Guía específica del Grupo de Información en Reproducción Elegida $(\mathrm{GIRE})^{\prime 27}$ fue elaborada al terminar las etapas de organización referentes a la identificación, clasificación, ordenación y guarda. Los elementos que conforman este instrumento fueron tomados del "Manual de procedimientos técnicos del AHUNAM", y a continuación los enumero:

1. Código de referencia y clave del fondo o colección documental.

2. Nombre completo del fondo o colección.

3. Fecha en que se generaron los documentos.

4. Volumen y soporte de los documentos.

5. Nombre de quien produjo o reunió los documentos.

6. Historia de la institución o reseña biográfica de quien generó o reunió los documentos.

7. Historia archivística del fondo o colección.

8. Forma de ingreso.

9. Resumen del contenido del fondo o colección.

10. Valoración, selección y eliminación.

11. Organización, especificar el tipo de clasificación y ordenación.

12. Condiciones de reproducción.

13. Idioma(s) y escritura(s) en que viene consignada la información.

14. Características físicas de los documentos.

15. Instrumentos de descripción.

16. Notas u observaciones. ${ }^{28}$

En general, el material está en buen estado y puede ser consultado por los usuarios; ya puede darse el servicio con la "Guía del orden como llegó el fondo Grupo de Información en Reproducción Elegida (GIRE) al AHUNAM. Relación de Ordenación y Guarda de documentos, en caja y expediente", 29 instrumento ela-

\footnotetext{
${ }^{26}$ Flores Padilla, "Manual de procedimientos técnicos...", 70.

27 Ángel Ángeles, "Guía específica del Grupo de Información en Reproducción Elegida (GIRE)" (México: UNAM, IISUE, AHUNAM), 36 (inédita).

28 lbid., 70-73.

29 Ángel Ángeles, "Guía del orden como llegó el fondo Grupo de Información en Reproducción Elegida (GIRE) al AHUNAM. Relación de Ordenación y Guarda de documentos, en caja y expediente", 4 vols. (México: UNAM, IISUE, AHUNAM, 2018), 1581 (inédita).
} 
borado para dejar un registro del movimiento que se hizo en la documentación y saber en qué caja y expediente se encuentra cada documento. Esta guía facilitó la consulta en tanto se elaboraba el catálogo por expediente, la relación del fondo GIRE y el catálogo por documento de las cajas 202 a 212, los cuales ya están concluidos y pueden solicitarse en el AHUNAM.

\section{La foliación y la descripción. Catálogo por expediente}

Estas dos etapas también están terminadas. El Manual de procedimientos técnicos para archivos menciona que la foliación "es la numeración de todas y cada una de las fojas que conforman el grupo documental, y podrá hacerse por fondo, sección, subsección, serie o expediente, atendiendo a los requerimientos prácticos del archivo". 30

En el caso del fondo GIRE, la foliación se hizo colocando el número en la parte superior derecha por expediente, es decir se colocó 1, 2, 3... consecutivamente hasta cubrir todas las fojas del expediente 1, para continuar con el expediente 2, folio 1, 2, 3... hasta cubrir todas sus fojas. Esta forma de foliar por expediente es de gran ayuda en caso de que se traspapele una foja o se integren nuevos documentos. La foliación es importante para que los documentos no sean desordenados por los usuarios o por el propio archivista, y permite reordenarlos nuevamente, así como llevar un control de las fojas.

La siguiente etapa es la descripción, para la cual podemos considerar lo que menciona Cruz Mundet:

La descripción de los documentos constituye la parte culminante del trabajo archivístico y viene a coincidir exactamente en su finalidad con la de la propia documentación: informar. Si ésta es clasificada, ordenada... en definitiva, conservada, es porque contiene información; si existen los archivos y sus profesionales no es por la mera complacencia de guardar objetos por valiosos que sean, sino por la necesidad de obtener información precisa en distintos momentos y para múltiples finalidades. Es una tarea primordial del quehacer archivístico y una consecuencia ineludible de la organización de los fondos, encaminada a poner los documentos en servicio, es decir, hacer de ellos un útil disponible para la sociedad. ${ }^{31}$

\footnotetext{
${ }^{30}$ Villanueva, Manual de procedimientos técnicos para archivos, 79.

${ }^{31}$ Cruz Mundet, Manual de archivística, 255.
} 
Para la descripción se elaboró un catálogo por expediente, dado que se trata de:

Un instrumento que describe el contenido de todas y cada una de las unidades documentales compuestas (expedientes, registros, dossiers) o simples (piezas documentales), que integran una serie, una sección, un fondo o colección.

Su objetivo es servir como medida de control y como auxiliar en la localización precisa de la información que se requiere y orienta al usuario sobre el contenido de los documentos, señalando sus características particulares. ${ }^{32}$

El catálogo por expediente contiene una introducción con información acerca de la creación del GIRE y la donación de su fondo al AHUNAM, del proceso de organización por medio de las seis etapas descritas en este trabajo, así como las fichas catalográficas que se están elaborando por expediente y que cuentan con los siguientes elementos:

\section{Artículos, folletos y hemerografía: ${ }^{33}$}

1. Núm. de ficha

2. Años

3. Lugar

4. Entrada descriptiva

5. Observaciones

6. Fondo

7. Sección

8. Serie

9. Caja

10. Expediente

11. Fojas

12. Folios

\footnotetext{
${ }^{32}$ Flores Padilla, "Manual de procedimientos técnicos...", 75.

${ }^{33}$ Los elementos de las fichas catalográficas están más completos porque se cuenta con más información, y casi finalizan las etapas de la organización de un fondo documental. Es importante mencionar que se elaborará un catálogo por expediente, pero dentro de la descripción encontraremos el catálogo por documento, que es una descripción aún más particular, la cual no realizaremos en este procedimiento de organización documental.
} 


\section{Revistas:}

1. Núm. de ficha

2. Años

3. Lugar

4. Título

5. ISSN

6. Núm. de fascículos

7. Temáticas

8. Observaciones

9. Fondo

10. Sección

11. Serie

12. Caja

13. Expediente

14. Fojas

15. Folios o páginas

\section{Objetos:}

1. Núm. de ficha

2. Año

3. Lugar

4. Título

5. Imagen (digitalización)

6. Entrada descriptiva

7. Observaciones

8. Fondo

9. Sección

10. Serie

11. Caja

12. Expediente

13. Núm. de piezas

14. Folios 
Se digitalizó el material para ilustrar la ficha de los objetos y carteles.

\section{Carteles:}

1. Núm. de ficha

2. Año

3. Lugar

4. Título

5. Imagen (digitalización)

6. Entrada descriptiva

7. Observaciones

8. Medidas

9. Fondo

10. Sección

11. Serie

12. Caja

13. Expediente

14. Fojas

15. Folios

La organización del fondo documental GIRE concluyó en agosto del 2019, fue foliado todo el material y con la descripción se obtuvieron los siguientes instrumentos de consulta: un catálogo por expediente y otro por documento (cajas 202-212), así como una relación donde una tabla describe las siguientes columnas: cajas, secciones, series, expedientes, años, documentos, fojas, folios y páginas, porque el fondo cuenta con 558 títulos de revistas.

\section{Conclusiones}

La organización del fondo documental del Grupo de Información en Reproducción Elegida GIRE ha concluido y ya puede ser consultado y reproducido. El método de organización que se siguió es el planteado por Mariano Mercado en su artículo "Guía del fondo Heberto Castillo Martínez", donde aborda las seis etapas de identificación, clasificación, ordenación, guarda, foliación y descripción.

La ventaja de este método es que permite, desde el inicio, consultar los fondos documentales, gracias a que en la etapa de identificación y clasificación se cuenta con dos instrumentos de consulta, la "Guía del orden..." y el cuadro de 
clasificación. Esta guía también sirve para elaborar cualquier otro instrumento de consulta, llámese inventario, guía específica o catálogo por expediente.

Es importante mencionar que otros métodos de ordenación de fondos documentales no incluyen la "Guía del orden...", la cual sirve como registro y deja testimonio del estado del fondo documental a su llegada al repositorio. Asimismo, esa guía ayuda a que en poco tiempo pueda darse servicio a los usuarios, como ocurrió en el caso del fondo GIRE, cuya guía ya había sido elaborada y ello permitió que la documentación no se moviera simultáneamente y provocara un caos.

Este artículo busca difundir la existencia del fondo GIRE, que para facilitar su consulta ya cuenta con los instrumentos mencionados. Para revisarlos, pueden ser solicitados en la sala de consulta del AHUNAM, que se encuentra en el segundo piso de la Hemeroteca Nacional de México.

\section{Referencias}

Ángeles Fernández, Ángel. "Catálogo por documento del Grupo de Información en Reproducción Elegida (GIRE)". México: Universidad Nacional Autónoma de México, Instituto de Investigaciones sobre la Universidad y la Educación, Archivo Histórico, 2019. Inédito.

Ángeles Fernández, Ángel. "Catálogo por expediente del Grupo de Información en Reproducción Elegida (GIRE)". México: Universidad Nacional Autónoma de México, Instituto de Investigaciones sobre la Universidad y la Educación, Archivo Histórico, 2019. Inédito.

Ángeles Fernández, Ángel. "Guía del orden como llegó el fondo Grupo de Información en Reproducción Elegida (GIRE). Relación de Ordenación y Guarda de documentos, en caja y expediente", 4 vols. México: Universidad Nacional Autónoma de México, Instituto de Investigaciones sobre la Universidad y la Educación, Archivo Histórico, 2018. Inédito.

Ángeles Fernández, Ángel. "Guía del orden en el cual ingresó el fondo Grupo de Información en Reproducción Elegida al AHUNAM", 4 vols. México: Universidad Nacional Autónoma de México, Instituto de Investigaciones sobre la Universidad y la Educación, Archivo Histórico, 2017. Inédito.

Ángeles Fernández, Ángel. "Guía específica del Grupo de Información en Reproducción Elegida (GIRE)". México: Universidad Nacional Autónoma de México, Instituto de Investigaciones sobre la Universidad y la Educación, Archivo Histórico. Inédito. 
Ángeles Fernández, Ángel. "Relación del Grupo de Información en Reproducción Elegida (GIRE)". México: Universidad Nacional Autónoma de México, Instituto de Investigaciones sobre la Universidad y la Educación, Archivo Histórico, 2019. Inédito.

Archivo General de la Nación (México). Instructivo para elaborar el Cuadro general de clasificación archivística. México: AGN, 2012.

Cruz Mundet, José Ramón. Manual de archivística. España: Fundación Germán Sánchez Ruipérez, 2003.

Flores Padilla, Georgina, coord. "Manual de procedimientos técnicos del Archivo Histórico de la UNAM". México: Universidad Nacional Autónoma de México, Instituto de Investigaciones sobre la Universidad y la Educación, Archivo Histórico [s. a.]. Inédito.

Grupo de Información en Reproducción Elegida. Boletín Trimestral sobre Reproducción Elegida, núm. 1 (abril de 1994).

Grupo de Información en Reproducción Elegida. Acceso el 2 de diciembre de 2019. https://www.gire.org.mx/quienes-somos.

Instituto Nacional de Transparencia, Acceso a la Información y Protección de Datos Personales. Ley General de Archivos. México: INAl, 2019.

Mercado Estrada, Mariano. "Guía del fondo Heberto Castillo Martínez". En Teoría y práctica archivística III. Coordinación de Mariano Mercado Estrada. México: Universidad Nacional Autónoma de México, Centro de Estudios sobre la Universidad, 2003.

Mondragón Aguilar, María Sandra. "Descripción de la Correspondencia de las Obras Literarias del Fondo Francisco L. Urquizo". En Teoría y práctica archivística VI. Coordinación de Gustavo Villanueva. Cuadernos del Archivo Histórico de la UNAM 22. México: Universidad Nacional Autónoma de México, Instituto de Investigaciones sobre la Universidad y la Educación, 2012.

Norma Internacional General de Descripción Archivística ISAD(G). Madrid: Consejo Internacional de Archivos, 2000. Acceso el 2 de diciembre de 2019. http://www.ica.org/?lid=10207.

Oficio dirigido al director del Instituto de Investigaciones sobre la Universidad y la Educación, dr. Mario Rueda Beltrán, firmado por la mtra. Regina Tamés Noriega, directora del Grupo de Información en Reproducción Elegida, A. C., GIRE, con fecha del 29 de abril de 2016.

Oficio de aceptación del fondo documental GIRE, firmado por la dra. Clara Ramírez, coordinadora AHUNAM-IISUE, con fecha del 2 de mayo de 2016. 


\begin{abstract}
Villanueva Bazán, Gustavo, coord. Manual de procedimientos técnicos para archivos históricos de universidades e instituciones de educación superior. México: Universidad Nacional Autónoma de México, Centro de Estudios sobre la Universidad / Archivo Histórico de la Benemérita Universidad Autónoma de Puebla, 2002.
\end{abstract}

